\title{
Prospect of GNR as Channel Material for Tunnel Field Effect Transistor
}

\author{
Jayabrata Goswami, Anuva Ganguly, Anirudhha Ghosal and J.P. Banerjee
}

\begin{abstract}
The design and simulation of a P-Channel Tunnel Field Effect Transistors (TFETs) with Graphene Nanoribbon (GNR) as channel material is carried out to achieve low sub-threshold swing and high onoff current ratio from the device. A self-consistent iterative method is used to solve one dimensional Poisson equation subject to appropriate boundary conditions and obtain the energy band diagram. The drain current is calculated from the energy dependent tunneling probability and Fermi functions at the source and drain regions. The channel length, ribbon width and gate oxide thickness of the device are appropriately designed to achieve high performance from the device. It is observed that the optimized device provides high ON-OFF current ratio $\left(6.5 \times 10_{4}\right)$ and low sub-threshold swing (8mV/decade), for a channel length of $20 \mathrm{~nm}$ and channel width of $4 \mathrm{~nm}$.
\end{abstract}

\section{Keywords}

GNR TFET, Sub-threshold swing, ON/OFF Current ratio

\section{INTRODUCTION}

Fundamental physical limitation of Silicon MOSFETs in Ultra Large Scale or Giga Scale Integration has led to the proposal of some novel non-classical transistors such as Tunnel Field Effect Transistors (TFETs) as future replacement of $\mathrm{Si}$ MOSFETs. In recent years TFETs are attracting the attention of researchers for low-voltage and low power application. The operation of TFETs is primarily dependent on band to band tunnelling (BTBT) of carriers from source and drain to the channel region.

It is reported [1-2] that the magnitude of SS of TFET can be brought down the thermionic limit of $60 \mathrm{mV} /$ decade at room temperature. Tunnel FETs based on lower band gap semiconductor provide higher ON-state current than those based on higher band gap semiconductor. Thus the $\mathrm{ON}$-state current in Ge TFETs is higher than that in Si TFETs. The off-current is however higher in lower band gap such as Ge than in higher band gap, Si. This leads to a degradation of ON-OFF current ratio in Ge TFETs degrading its performance [3].

TFETs with carbon based channel material are reported to excel conventional TFETs as regards subthreshold swing and ON-OFF current ratio. Leem et.al [4] reported that the carbon nanotube heterojunction tunnel field effect transistors(CNTTFET) provide lower subthershold swing in the range of $22-26 \mathrm{mV} /$ decade at a supply voltage $0.2 \mathrm{~V}$ at channel length $15 \mathrm{~nm}$ with source drain extension is $10 \mathrm{~nm}$ and oxide thickness is $1.7 \mathrm{~nm}$. TFETs based on Graphene Nanoribbon(GNR) have been proposed in recent years which show excellent performance of these devices as regards lower values of SS and higher ON-OFF current ratio.

Graphene possesses some favourable electronic and physical properties as channel material of TFETs. The electron mobility in Graphene is very high of the order of $105 \mathrm{~cm}_{2} / \mathrm{V}$-sec [5]. Further the bandgap of GNR can be engineered by varying its ribbon width. It has robust mechanical strength and high thermal conductivity with atomic configuration at the ribbon edge. It exhibits symmetric dispersion relation for both electrons and holes and is less sensitive to doping fluctuation.

Fiori et.al [6] reported that ON-OFF current ratio is more than 103 with SS lower than $20 \mathrm{mV} /$ decade at a supply voltage $0.1 \mathrm{~V}$ from a double gate bilayer GNR TFET at channel length 40nm.

The purpose of the present paper is to present a semi-analytical model of a GNR PTFET and analysis of its performance parameters through appropriate design of device structural parameters to realize low value of SS and high value of current ratio at low gate voltage.

II. Device Structure AND SElF-CONSISTENT MODEL FOR NUMERICAL SIMULATION OF GNR PTFET

The quasi one dimensional geometry of $\mathrm{p}+$ channel GNR TFET device structure is shown in Figure 1. The $n+$ source, $p+$ channel and $p+d r a i n$ regions are heavily doped with doping concentration by chemical or electrostatic doping process. The doping concentrations of $\mathrm{n}+$ region and $\mathrm{p}+$ regions are taken to be $3.4 \times 1020 \mathrm{~cm}-3$ and $1.1 \times 1020 \mathrm{~cm}-3$ respectively. A monolayer graphene having thickness (tGNR) of the order of few Angstrom i.e. 3-4A0 is deposited over the Si substrate which acts as channel material [7]. A thin layer of high-k dielectric material like $\mathrm{Y}_{2} \mathrm{O}_{3}\left(\varepsilon_{\mathrm{r}}=16\right)$ having a thickness $\left(\mathrm{t}_{\mathrm{ox}}\right)$ of $2 \mathrm{~nm}$ is incorporated in between the gate and GNR.

The channel is assumed to be fully depleted in the OFF state at zero gate voltage. Both the gate and drain are reverse biased with respect to source. The reverse bias gate - source and drain - source voltages are $\mathrm{V}_{\mathrm{GS}}$ and $\mathrm{V}_{\mathrm{DS}}$ respectively. The $\mathrm{p}+$ channel is taken in positive $\mathrm{x}$-direction whose length is $\mathrm{L}_{\mathrm{CH}}$. 


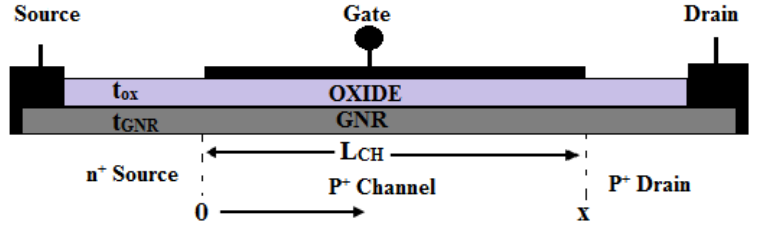

Fig. 1.The structure of a $\mathrm{p}^{+}$-channel GNR TFET

The surface potential at any position in the channel region of GNR PTFET can be obtained from numerical solution of one-dimensional Poisson's equation [8] subject to the appropriate boundary conditions.

$$
\frac{\mathrm{d}^{2} \varphi_{\text {surf }}(\mathrm{x})}{\mathrm{dx}^{2}}-\frac{\varphi_{\text {surf }}(\mathrm{x})-\mathrm{V}_{\mathrm{GS}}-\mathrm{V}_{\mathrm{BI}}}{\lambda^{2}}=-\frac{\mathrm{q} \rho(\mathrm{x})}{\varepsilon_{\mathrm{GNR}}}
$$

Where $\mathrm{V}_{\mathrm{GS}}$ is the gate to source potential, $\mathrm{V}_{\mathrm{BI}}$ is the built-in potential, $\varphi_{\text {surf }}(\mathrm{x})$ is the surface potential at position $\mathrm{x}, \lambda$ is the screening length for the particular device structure, $\rho(x)$ is the total charge density and $\varepsilon_{\mathrm{GNR}}$ is the permittivity of GNR. The total charge density is approximately equal to the impurity charge density since the source and drain regions are highly doped and channel is assumed to be fully depleted at zero gate bias.

Graphene nanoribbon is obtained by cutting two dimensional graphene sheets in narrow stripes which leads to lateral confinement and induces a bandgap. GNR possesses the unique property of width tunable band gap.

The band gap energy of GNR is related to ribbon width as follows:

$$
\xi_{\mathrm{G}}=\xi_{\mathrm{C}}-\xi_{\mathrm{V}}=\xi_{\mathrm{S}=+1}-\xi_{\mathrm{S}=-1}=\frac{2 \pi \hbar v_{\mathrm{F}}}{3 \mathrm{w}_{\mathrm{GNR}}}
$$

Here the width of GNR is much less than its length. Thus the bandgap of GNR depends on the ribbon width $\mathrm{w}_{\mathrm{GNR}}$ and $\left(v_{F}\right)$ Fermi velocity.

A screening length $\lambda$ appears in Poisson's given by [9]

$$
\lambda=\sqrt{\left(\frac{\varepsilon_{\mathrm{GNR}}}{\varepsilon_{\mathrm{OX}}}\right) \mathrm{t}_{\mathrm{GNR}} \mathrm{t}_{\mathrm{ox}}}=\sqrt{\mathrm{t}_{\mathrm{GNR}} \mathrm{t}_{\mathrm{ox}}}
$$

In the above expressions, $\mathrm{t}_{\mathrm{GNR}}$ and $\mathrm{t}_{\mathrm{ox}}$ are the thickness of GNR and gate oxide respectively. The permittivities of GNR and gate-oxide are $\varepsilon_{\mathrm{GNR}}$ and $\varepsilon_{\mathrm{OX}}$ respectively. A high-k dielectric material
$\left(\mathrm{Y}_{2} \mathrm{O}_{3}\right)$ having a relative permittivity of 16 is used in the analysis. The high-k dielectric material like $\mathrm{Y}_{2} \mathrm{O}_{3}$ is used to reduce the leakage current from gate to channel. The relative permittivity of GNR is approximated to be same as that of high-k dielectric for simplicity of analysis so that the screening length becomes permittivity independent.

The performance parameters of GNR PTFET are optimized by varying the channel length, ribbon width and gate oxide thickness of the device.

The energy band profile of GNR PTFET is obtained from self-consistent numerical solution of quasi onedimensional Poisson's equation in source, channel and drain regions subject to the following boundary conditions:

(a)The electric field falls to zero at the highly doped source and drain ends of the channel.

(b) Both the electric field and potential are continuous at the source-channel and drain-channel interfaces.

(c) The separation of Fermi energy level from both the top of the conduction band and the bottom of the valance band are taken to be equal to the thermal energy of the carrier.

(d) At zero gate bias the valence band in the channel is coincident with the Fermi level at the source end.

The surface potential and barrier height can be extracted from the energy band diagram

At the starting point of iterations, the tunneling injected charges from source to channel $\left(Q_{C S}\right)$, drain to channel $\left(\mathrm{Q}_{\mathrm{CD}}\right)$ are taken to be zero. Therefore total channel charge $\left(Q_{C}=Q_{C S}+Q_{C D}=0\right)$ is also zero.

The expression of tunneling probability as a function of energy is given by

$$
\mathrm{T}_{\mathrm{S}}(\xi)=\exp \left(-2 \int\left|\mathrm{k}_{\mathrm{x}}\right| \mathrm{dx}\right)
$$

The expression of the imaginary wave vector is given by

$$
k_{x}=\sqrt{\left[\xi_{\mathrm{c}}(\mathrm{x})-\xi_{-}-\xi_{\mathrm{G}} / 2\right]^{2}-\left(\xi_{\mathrm{G}} / 2\right)^{2}}
$$

The wave vector, $\mathrm{k}_{\mathrm{x}}$ is imaginary so that the tunneling probability becomes an exponentially attenuated function and correspondingly the tunneling current wave decays exponentially in the channel. If $\mathrm{k}_{\mathrm{x}}$ is real then the tunneling wave will be a propagating one which is not allowed.

Using equation (5), equation (4) can be written as 


$$
\mathrm{T}_{\mathrm{S}}(\xi)=\exp \left(-\frac{2}{\hbar v_{\mathrm{F}}} \int_{\mathrm{x}_{\mathrm{i}}}^{\mathrm{x}_{\mathrm{f}}} \sqrt{\left(\xi_{\mathrm{G}} / 2\right)^{2}-\left(\xi_{\mathrm{C}}(\mathrm{x})-\left(\xi+\xi_{G} / 2\right)^{2}\right)} \mathrm{dx}\right)
$$

Where $x_{i}$ and $x_{f}$ are the initial and final positions of tunneling respectively and $\xi_{\mathrm{C}}(\mathrm{x})$ is the energy at the bottom of conduction band.

The expressions for $\mathrm{Q}_{\mathrm{CS}}$ and $\mathrm{Q}_{\mathrm{CD}}$ are given by[10]

$$
\begin{gathered}
\mathrm{Q}_{\mathrm{CS}}=-\mathrm{q} \int \rho_{\mathrm{GNR}}(\xi) \mathrm{T}_{\mathrm{S}}(\xi) \mathrm{d} \xi \\
\mathrm{Q}_{\mathrm{CD}}=\mathrm{q} \int \rho_{\mathrm{GNR}}(\xi)\left[f_{\mathrm{D}}(\xi)\left(\frac{2}{\mathrm{~T}_{\mathrm{S}}(\xi)}-1\right)\right] \mathrm{d} \xi
\end{gathered}
$$

In equations (7) and (8), $\rho_{\mathrm{GNR}}$ is the density of states function for GNR given by

$$
\rho_{\mathrm{GNR}}=\frac{1}{\pi \hbar v_{\mathrm{f}}} \frac{\xi}{\sqrt{\xi^{2}-\left(\xi_{\mathrm{G}} / 2\right)^{2}}}
$$

where $\xi>\xi_{\mathrm{G}} / 2$ and $\xi_{\mathrm{G}}$ is the band gap of GNR, $f_{S}$ and $f_{D}$ denote the Fermi-Dirac distribution functions at the source and the drain respectively, $v_{\mathrm{F}}$ is the Fermi velocity $\left(10^{6} \mathrm{~m} / \mathrm{sec}\right)$ and $T_{S}(\xi)$ is the energy dependent tunneling probability.

Substituting the expression of $\rho_{\mathrm{GNR}}$ and $\mathrm{T}_{\mathrm{S}}(\xi)$ from equations (9) and (6) respectively in equations (7) and (8), numerical integration is carried out to calculate $\mathrm{Q}_{\mathrm{CS}}$ and $\mathrm{Q}_{\mathrm{CD}}$.

The tunneling current from the source to drain is obtained from Landauer formula given below [11].

$$
\mathrm{I}_{\mathrm{D}}=\frac{\mathrm{q}}{\pi \hbar} \int\left(f_{\mathrm{D}}(\xi)-f_{\mathrm{S}}(\xi)\right) \mathrm{T}_{\mathrm{S}}(\xi) \mathrm{d} \xi
$$

Numerical integration of the product of tunneling probability, $\mathrm{T}_{\mathrm{s}}(\xi)$ and the difference between the Fermi-Dirac distribution functions at the source and drain regions is carried out to obtain the drain current, $\mathrm{I}_{\mathrm{D}}$.

\section{Simulation Results}

The simulated energy band diagrams for the optimized GNR PTFET in the OFF state $\left(\mathrm{V}_{\mathrm{GS}}=0 \mathrm{~V}\right)$ and $\mathrm{ON}$ state $\left(\mathrm{V}_{\mathrm{GS}}=-0.1 \mathrm{~V}, \mathrm{~V}_{\mathrm{DS}}=-0.1 \mathrm{~V}\right)$ are shown in Figs. 2(a) and 2(b) respectively corresponding to the values of $\mathrm{w}_{\mathrm{GNR}}$ and $\mathrm{L}_{\mathrm{CH}}$ as $4 \mathrm{~nm}$ and $20 \mathrm{~nm}$ respectively. The Fermi energy levels $\left(E_{F}\right)$ in the band diagrams for both the OFF and ON states of the device are also shown. The band-to-band tunneling in TFET depends on the variation of position of the energy band in the channel region with respect to that in the source and drain regions. Fig. 2(b) shows that the applied gate voltage pulls up the energy band in the channel region in the $\mathrm{ON}$ state with respect to that in the OFF state which gives rise to tunneling current.

The OFF current for long channel GNR $\left(\mathrm{L}_{\mathrm{CH}}=\right.$ 20nm) arises from thermionic emission over the barrier only and direct source to drain tunneling is negligibly small. The OFF current in GNR is calculated from the following equation [12].

$$
\mathrm{I}_{\mathrm{OFF}}=\left[\mathrm{q}^{2} \mathrm{~V}_{\mathrm{T}} / \hbar \pi\right] \exp \left(-\mathrm{qV}_{\mathrm{B}} / \mathrm{qV}_{\mathrm{T}}\right)
$$

re $\mathrm{qV}_{\mathrm{B}}$ is the barrier height and $\mathrm{qV}_{T}$ is the thermal energy.

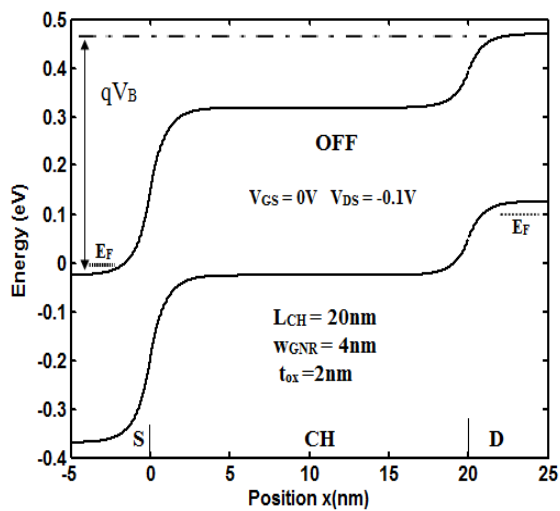

(a)

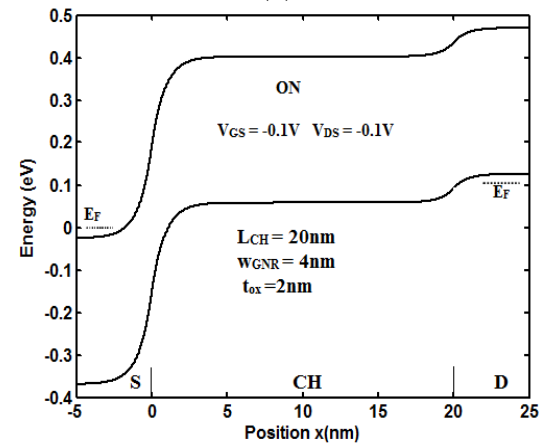

(b)

Fig. 2. Energy band diagrams for a GNR PTFET in the (a) OFF-state and (b) ON-state.

The OFF current in GNR PTFET is $0.24 \mathrm{pAfor}$ a ribbon width of $4 \mathrm{~nm}$ and channel length $20 \mathrm{~nm}$ respectively.

Figure 3(a) shows the ON current versus gate to source bias $\left(\mathrm{V}_{\mathrm{GS}}\right)$ for channel length of $20 \mathrm{~nm}$ and fixed ribbon width of $4 \mathrm{~nm}$ and oxide thickness of $2 \mathrm{~nm}$. The On state current is found $1.58 \mu \mathrm{A}$. Fig 3(b) shows the ON-OFF current ratio versus gate to source voltage $\left(\mathrm{V}_{\mathrm{GS}}\right)$. The $\mathrm{ON}-\mathrm{OFF}$ current ratio is found $6.5 \times 10^{4}$ at $\mathrm{V}_{\mathrm{GS}}=-0.1 \mathrm{~V}$. 


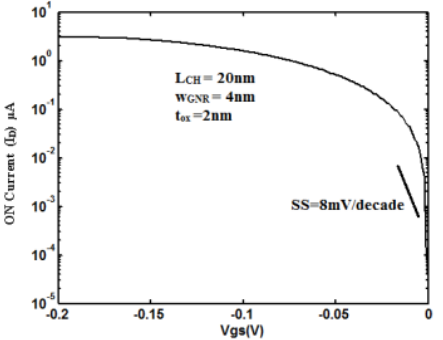

(a)

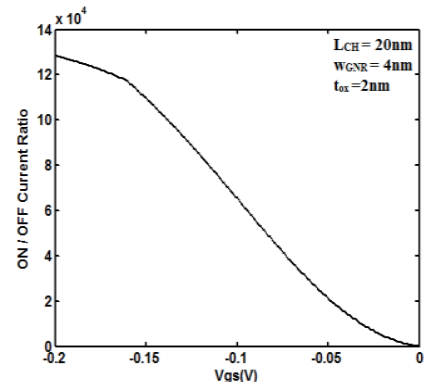

(b)

Fig. 3. (a) ON current versus $V_{G S}$ of GNR PTFET (b) ON/OFF current ratio versus $V_{G S}$ of GNR PTFET.

The sub-threshold swing (SS) of a Tunnel FieldEffect transistor is related to the transconductance( $g_{m}$ ) and sub-threshold drain current $\left(I_{D}\right)$ as follows [13]:

$$
\mathrm{SS}=\ln 10\left(\frac{\mathrm{g}_{\mathrm{m}}}{\mathrm{I}_{\mathrm{D}}}\right)
$$

The sub-threshold slope (SS) of the optimized GNR PTFET is found to be $8 \mathrm{mV} /$ decade at $\mathrm{V}_{\mathrm{GS}}=$ $0.1 \mathrm{~V}$.The device shows good switching property due to very low OFF state current and very high value of ON state current. Thus the optimized GNR PTFET will be very much suitable for low power digital application with high ON-OFF current ratio.

\section{CONCLUSION}

A p+ channel GNR Tunnel Field Effect Transistor has been designed for optimum performance with respect to higher ON-OFF current ratio and lower subthreshold swing. The values of $\mathrm{I}_{\mathrm{ON}} / \mathrm{I}_{\mathrm{OFF}}$ and SS for the optimized device are $6.5 \times 10^{4}$ and $8 \mathrm{mV} /$ decade respectively when channel width and length are designed at $4 \mathrm{~nm}$ and $20 \mathrm{~nm}$ respectively. The results show that the optimized GNR PTFET will be useful for low power digital application.

\section{REFERENCES}

[1] Appenzeller, J., Lin, Y. M., Knoch, J., \& Avouris, P. (2004). Band-to-band tunneling in carbon nanotube field-effect transistors. Physical review letters, 93(19), 196805.

[2] Wang, P. F., Hilsenbeck, K., Nirschl, T., Oswald, M., Stepper, C., Weis, M., \& Hansch, W. (2004). Complementary tunneling transistor for low power application. Solid-State Electronics, 48(12), 22812286.

[3] Toh, E. H., Wang, G. H., Samudra, G., \& Yeo, Y. C. (2008). Device physics and design of germanium tunneling field-effect transistor with source and drain engineering for low power and high performance applications. Journal of Applied Physics, 103(10), 104504.

[4] Leem, L., Srivastava, A., Li, S., Magyari-Köpe, B., Iannaccone, G., Harris, J. S., \& Fiori, G. (2010, December). Multi-scale simulation of partially unzipped CNT hetero-junction tunneling field effect transistor. In Electron Devices Meeting (IEDM), 2010 IEEE International (pp. 32-5). IEEE.

[5] Morozov, S. V., Novoselov, K. S., Katsnelson, M. I., Schedin, F., Elias, D. C., Jaszczak, J. A., \& Geim, A. K. (2008). Giant intrinsic carrier mobilities in graphene and its bilayer. Physical review letters, 100(1), 016602.

[6] Fiori, G., \& Iannaccone, G. (2009). Ultralow-voltage bilayer graphene tunnel FET. IEEE Electron Device Letters, 30(10), 1096-1098.

[7] Fang, J., Vandenberghe, W. G., \& Fischetti, M. V. (2016). Microscopic dielectric permittivities of graphene nanoribbons and graphene. Physical Review B, 94(4), 045318.

[8] Appenzeller, J., Knoch, J., Bjork, M. T., Riel, H., Schmid, H., \& Riess, W. (2008). Toward nanowire electronics. IEEE Transactions on electron devices, 55(11), 2827-2845.

[9] Yan, R. H., Ourmazd, A., \& Lee, K. F. (1992). Scaling the Si MOSFET: From bulk to SOI to bulk. IEEE Transactions on Electron Devices, 39(7), 1704 1710.

[10] Yan, R. H., Ourmazd, A., \& Lee, K. F. (1992). Scaling the Si MOSFET: From bulk to SOI to bulk. IEEE Transactions on Electron Devices, 39(7), 1704 1710.

[11] Fahad, M. S., Srivastava, A., Sharma, A. K., \& Mayberry, C. (2016). Analytical current transport modeling of graphene nanoribbon tunnel field-effect transistors for digital circuit design. IEEE Transactions on Nanotechnology, 15(1), 39-50.

[12] Sze, S. M. (1981). Physics of semiconductor devices. New York: John Wi-ley \& Sons.

[13] Barboni, L., Siniscalchi, M., \& Sensale-Rodriguez, B. (2015). TFET-based circuit design using the transconductance generation efficiency gm/Id Method method. IEEE Journal of the Electron Devices Society, 3(3), 208-216.

Jayabrata Goswami

e-mail:goswamijayabrata@gmail.com

Anuva Ganguly

e-mail:gangulyanuva@gmail.com

Anirudhha Ghosal

e-mail: aghosal2008@gmail.com

J.P. Banerjee

e-mail:scope.jcb@gmail.com

Institute of Radio Physics and Electronics, University of Calcutta, Kolkata, India 